hep-th/0001218

\title{
BFV-BRST analysis of equivalence between noncommutative and ordinary gauge theories
}

\author{
Ömer F. DAYI \\ Physics Department, Faculty of Science and Letters, Istanbul Technical University, 80626 Maslak, \\ Istanbul, Turkey \\ and \\ Feza Gürsey Institute, P.O.Box 6, 81220 Çengelköy, Istanbul, Turkey. I
}

Constrained hamiltonian structure of noncommutative gauge theory for the gauge group $U(1)$ is discussed. Constraints are shown to be first class, although, they do not give an Abelian algebra in terms of Poisson brackets. The related BFV-BRST charge gives a vanishing generalized Poisson bracket by itself due to the associativity of $*$-product. Equivalence of noncommutative and ordinary gauge theories is formulated in generalized phase space by using BFV-BRST charge and a solution is obtained. Gauge fixing is discussed.

\footnotetext{
${ }^{1}$ E-mails: dayi@itu.edu.tr and dayi@gursey.gov.tr.
} 
Noncommutative geometry is one of the most attractive subjects of physics and mathematics [1]. Attention to it is increased considerably after the observation that in string theories noncommutativity of space appears in a natural way [2]. Seiberg and Witten used the ideas about the noncommutativity in string theory and showed that noncommutative and ordinary gauge theories are equivalent [3]. Following them there appeared several works on noncommutative gauge theories[国]. BRST symmetry of a noncommutative gauge theory in first order Lagrangian formulation was discussed in [5].

When one deals with a gauge theory, Lagrangian framework is suitable for perturbative calculations. However, understanding its Hamiltonian structure is essential to perform canonical quantization which can be used to derive some features like the correct measure of path integral and physical states.

In Hamiltonian framework, an ordinary gauge theory action leads to first class constraints which decrease the number of linearly independent phase space variables. For keeping track of gauge invariance, instead of getting rid of the unphysical degrees of freedom, one enlarges the phase space by introducing ghost fields possessing the opposite statistics of the constraints and write the BVF-BRST charge [6]. Now, invariance of the theory under the transformation of variables generated by the constraints (reminiscent of gauge invariance) replaced by the invariance under the transformations generated by the BFV-BRST charge. Action and measure of the related path integrals should be invariant under the BFV-BRST transformations. Once the canonical commutation relations between phase space fields are imposed, they can be written in terms of their normal modes. Thus, one can find the quantum BFV-BRST charge which is defined to be nilpotent and whose cohomology gives the physical states[7].

One expects that canonical formulation of noncommutative gauge theories can be studied in a similar manner, as far as the time coordinate is kept classical. We show that, indeed, this is the case, although, it was not guaranteed a priori: Gauge invariance of noncommutative gauge theory is not an invariance of the Lagrange density, in contrary to the ordinary case, but an invariance of the action,

Hamiltonian structure of ordinary gauge theories are well studied. Thus, we hope that showing the equivalence between ordinary and noncommutative gauge theories in terms of the generalized phase space variables will be useful in understanding features like canonical quantization of the latter.

Constrained hamiltonian structure of noncommutative gauge theory for the gauge group $U(1)$ is studied. This is the simplest gauge group, however, the main features of noncommuting gauge theories are already present. The related BFV-BRST charge which gives a vanishing generalized Poisson bracket with itself is presented. Equivalence of ordinary and noncommutative gauge theories is formulated in terms of generalized phase space variables and a solution is given. We also briefly discuss gauge fixing within this formalism. 
Noncommutative gauge theory for $U(1)$ in $\mathbb{R}^{d}$ is given with the action

$$
S=-\frac{1}{4} \int d^{d} x F_{\mu \nu} * F^{\mu \nu}
$$

in terms of the metric $\eta_{\mu \nu}=\operatorname{diag}(-1,1, \cdots)$, and

$$
F^{\mu \nu}(x)=\partial^{\mu} A^{\nu}-\partial^{\nu} A^{\mu}-i\left(A^{\mu} * A^{\nu}-A^{\nu} * A^{\mu}\right) .
$$

*-product is defined as

$$
G(x) * K(x)=G(x) e^{\frac{i}{2} \theta^{i j} \overleftarrow{\partial_{i}} \overrightarrow{\partial_{j}}} K(x)
$$

where $\theta^{i j}$ is an antisymmetric, constant matrix. The $*$-product reflects the fact that the space coordinates $x_{i}$ are noncommuting:

$$
x^{i} * x^{j}-x^{j} * x^{i}=i \theta^{i j} .
$$

(1) is invariant under the gauge transformations

$$
\delta A_{\mu}=\partial_{\mu} \lambda+i\left(\lambda * A_{\mu}-A_{\mu} * \lambda\right),
$$

because we have the equalities

$$
\begin{aligned}
\int d^{d-1} x G(x) *(K(x) * L(x)) & =\int d^{d-1} x G(x)(K(x) * L(x)) \\
& =\int d^{d-1} x(L(x) * G(x)) K(x), \\
& =\int d^{d-1} x(G(x) * K(x)) L(x),
\end{aligned}
$$

which follow from the assumption that all of the fields which we deal with are vanishing at infinity.

The time coordinate is not deformed and noncommutativity is due to the *product. Hence, the fields are ordinary ones and the canonical momenta are defined as usual:

$$
\begin{aligned}
P_{0} & =\frac{\partial S}{\partial \dot{A}^{0}}=0 \\
P_{i} & =\frac{\partial S}{\partial \dot{A}^{i}}=\partial_{0} A_{i}-\partial_{i} A_{0}-i\left(A_{0} * A_{i}-A_{i} * A_{0}\right) .
\end{aligned}
$$

The canonical hamiltonian reads

$$
H=\int d^{d-1} x\left(\frac{1}{2} P_{i}^{2}+\frac{1}{4} F_{i j} * F^{i j}-A_{0} \Phi(x)\right),
$$


where

$$
\Phi(x)=\partial_{i} P^{i}+i\left[P_{i}, A^{i}\right],
$$

written in terms of the Moyal bracket

$$
[G(x), K(x)] \equiv G(x) * K(x)-K(x) * G(x) .
$$

The primary constraints (3) should be constant in time:

$$
\left\{P_{0}(x), H\right\}=0
$$

where the basic Poisson brackets are

$$
\left\{P_{\mu}(x), A_{\nu}(y)\right\}=\eta_{\mu \nu} \delta(x-y)
$$

The condition (7) leads to the secondary constraints

$$
\Phi(x)=0
$$

Obviously, $P_{0}$ gives a vanishing Poisson bracket with $\Phi(x)$. To classify the constraints $\Phi(x)$ let us introduce a bosonic parameter $\lambda(x)$ and deal with

$$
\begin{aligned}
\Phi_{\lambda} & \equiv \int d^{d-1} x \Phi(x) \lambda(x) \\
& =\int d^{d-1} x \Phi(x) * \lambda(x) \\
& =\int d^{d-1} x P_{i}\left(-\partial_{i} \lambda(x)+i\left[P_{i}(x), \lambda(x)\right]\right) .
\end{aligned}
$$

The last equality follows from the fact that the Moyal bracket in (6) possesses only odd powers of $\theta^{i j}$. Poisson bracket of the integrated constraints is calculated:

$$
\left\{\Phi_{\lambda}, \Phi_{\kappa}\right\}=i \Phi_{[\lambda, \kappa]}
$$

where on the right hand side parameter is the Moyal bracket of the ones appearing on the left. Moreover, $\Phi(x)$ do not lead to new constraints:

$$
\left\{H_{0}, \Phi_{\lambda}\right\}=0
$$

where $H_{0}=\left.H\right|_{\Phi=0}$. Hence the whole set of constraints is given by (3) and (6) which are first class.

For the following discussions the primary constraints (3) are not essential. Hence, we do not deal with them any more by fixing the gauge as $A_{0}=0$ and setting $P_{0}(x)=0$. 
To perform the BFV-BRST analysis, we enlarge the phase space with the anticommuting ghost fileds $C(x)$ and their canonical conjugates $P_{C}(x)$ and use the generalized Poisson bracket

$$
\{G, K\}=\int d^{d-1} x\left(\frac{\partial_{r} G}{\partial \mathcal{P}(x)} \frac{\partial_{l} K}{\partial \mathcal{Q}(x)}-(-1)^{\eta(G) \eta(K)} \frac{\partial_{r} K}{\partial \mathcal{P}} \frac{\partial_{l} G}{\partial \mathcal{Q}}\right) .
$$

Here the subscripts $r$ and $l$ indicate right and left derivatives, $\eta(G)$ Grassmann parity of $G$ and $\mathcal{Q}, \mathcal{P}$ denote the generalized phase space coordinates and momenta including the original ones and the ghosts. We attribute also ghost numbers

$$
\operatorname{gh}(C)=-\operatorname{gh}\left(P_{C}\right)=1, \operatorname{gh}\left(A_{i}\right)=\operatorname{gh}\left(P_{i}\right)=0 .
$$

The BFV-BRST charge is

$$
\begin{aligned}
\Omega & =\int d^{d-1} x\left(\Phi(x) * C(x)-P_{C}(x)(C(x) * C(x))\right) \\
& =\int d^{d-1} x\left(\Phi(x) C(x)-\frac{1}{2}\left(P_{C}(x) * C(x)+C(x) * P_{C}\right) C(x)\right) .
\end{aligned}
$$

To deal with fermionic fields, we generalize the Moyal bracket as

$$
[G, K] \equiv G * K-(-)^{\eta(G) \eta(K)} K * G .
$$

Obviously, the generalized Moyal brackets satisfy the generalized Jacobi identity. Thus,

$$
\int d^{d-1} x(C * C)\left[P_{C}, C\right]=\frac{1}{3} \int d^{d-1} x\left(\left[[C, C], P_{C}\right]+\left[\left[C, P_{C}\right], C\right]+\left[\left[P_{C}, C\right], C\right]\right) C=0,
$$

which is the unique nontrivial term to conclude that the BFV-BRST charge satisfies

$$
\{\Omega, \Omega\}=0 .
$$

By introducing the rigid, fermionic parameter $\epsilon$ possessing ghost number -1 , the phase space fields transform as

$$
\begin{aligned}
\delta_{\epsilon} A_{i} & =-\epsilon\left(\partial_{i} C+i C * A_{i}-i A_{i} * C\right), \\
\delta_{\epsilon} C & =-i \epsilon C * C, \\
\delta_{\epsilon} P_{i} & =i \epsilon\left(P_{i} * C-C * P_{i}\right), \\
\delta_{\epsilon} P_{C} & =-\epsilon\left(\Phi+i P_{C} * C+i C * P_{C}\right),
\end{aligned}
$$

under the BFV-BRST charge (11).

Now, we would like to discuss the equivalence of noncommutative and ordinary gauge theories. Ordinary gauge theory action for the gauge group $U(1)$ is given in terms of the field strength $f_{\mu \nu}=\partial_{\mu} a_{\nu}-\partial_{\nu} a_{\mu}$, as

$$
S_{o}=\int d^{d} x f_{\mu \nu} f^{\mu \nu}
$$


The definition of canonical momenta $p_{\mu}$, yields the primary constraints $p_{0}(x)=0$. These lead to the secondary constraints $\partial_{i} p_{i}=0$, which are first class. We set strongly $p_{0}=0$ by fixing the gauge as $a_{0}=0$. Now, as usual we enlarge the phase space with the anticommuting ghost field $c(x)$ and its canonical conjugate $p_{c}(x)$, possessing ghost numbers 1 and -1 to write the BFV-BRST charge as

$$
\Omega^{o}=\int d^{d-1} x \partial_{i} p^{i} c
$$

Thus the BFV-BRST transformation of the fields is given as

$$
\begin{aligned}
\delta_{\epsilon}^{o} a_{i}=-\epsilon \partial_{i} c & , \quad \delta_{\epsilon}^{o} c=0 \\
\delta_{\epsilon}^{o} p_{i}=0 & , \quad \delta_{\epsilon}^{o} p_{c}=-\epsilon \partial_{i} p_{i} .
\end{aligned}
$$

Let us denote the phase space variables in a unified notation as

$$
Q_{z} \equiv\left(A_{i}, P_{i}, C, P_{C}\right), q_{z} \equiv\left(a_{i}, p_{i}, c, p_{c}\right)
$$

Then, we can formulate equivalence of the noncommutative and ordinary gauge theories as

$$
Q_{z}(q)+\delta_{\epsilon} Q_{z}(q)=Q_{z}\left(q+\delta_{\epsilon}^{o} q\right) .
$$

Moreover, there are the conditions

$$
\left.Q_{z}(q)\right|_{\theta=0}=q_{z}
$$

To first order in $\theta^{k l}$, there is a solution of (22)-(23):

$$
\begin{aligned}
A_{i}(a) & =a_{i}-\theta^{k l}\left(a_{k} \partial_{l} a_{i}-\frac{1}{2} a_{k} \partial_{i} a_{l}\right), \\
P_{i}(a, p) & =p_{i}-\theta^{k l} a_{k} \partial_{l} p_{i}, \\
C(a, c) & =c+\frac{1}{2} \theta^{k l} \partial_{k} c a_{l} \\
P_{C}\left(a, p, p_{c}\right) & =p_{c}+p_{c}\left(\partial_{j} p^{j}\right)^{-1} \theta^{k l} \partial_{k} p_{i} f_{i l}+\theta^{k l} \partial_{k} p_{c} a_{l} .
\end{aligned}
$$

As expected, the solutions $A_{i}(a)$ (24) and $C(a, c)$ (26) can be derived by using the solution for $A_{i}$ and the gauge parameter $\lambda$ given in [3]. At first glance (27) can be thought of being inconsistent with the constraint $\partial_{i} p^{i}=0$ of the ordinary $U(1)$ gauge theory. However, this is due to the fact that noncommutative and ordinary gauge groups can not be isomorphic to each other [3]: when one deals with the noncommutative gauge theory the constraint $\Phi=0$ leads to $\theta^{i j}=0$ for $\partial_{i} p^{i}=0$ and arbitrary gauge field $a_{i}$. There is another way of explaining this fact: observe that the BRST transformations of $P_{C}$ and $p_{c}(16),(20)$ are proportional to the related constraints, so that they are aware of the structure of the gauge group. Thus, as far as we deal with nonvanishing $\theta^{i j}$ we assume that $\partial_{i} p^{i} \neq 0$. 
For performing gauge fixing one enlarges the phase space with the bosonic and fermionic canonical conjugate pairs $(\Lambda, \Pi)$ and $\left(\bar{C}, \bar{P}_{C}\right)$ satisfying the generalized Poisson bracket relations

$$
\{\Lambda(x), \Pi(y)\}=\delta(x-y) \quad ; \quad\left\{\bar{C}(x), \bar{P}_{C}(y)\right\}=\delta(x-y) .
$$

$\Lambda, \Pi$ possess zero ghost number and

$$
\operatorname{gh}(\bar{C})=-\operatorname{gh}\left(\bar{P}_{C}\right)=-1
$$

Gauge fixed action which can be used in the related path integral can be given in terms of the gauge fixed hamiltonian

$$
H=H_{0}+\left\{\Psi, \Omega^{\prime}\right\}
$$

where $\Psi$ is the gauge fixing fermion possessing ghost number -1 and

$$
\Omega^{\prime}=\Omega+\int d^{d-1} x \Pi \bar{P}_{C}
$$

The easist choice for the gauge fixing fermion $\Psi$ is

$$
\Psi=\int d^{d-1}\left(P_{C} \xi+\bar{C} \Lambda\right)
$$

where $\xi$ is a function of the original fileds and defined to possess a nonvanishing Poisson bracket with $\Phi(x)$. The gauge fixed hamiltonian in this gauge becomes

$$
H=H_{0}+\int d^{d-1} x\left(C\{\Phi, \xi\} \bar{C}+\Lambda\left(\Phi+\left[C, P_{C}\right]\right)+\bar{P}_{C} P_{C}+\Pi \xi\right) .
$$

By choosing a gauge condition $\xi(x)$ we can perform perturbative calculations within the Hamiltonian approach to study noncommutative gauge theory and its relations with ordinary gauge theory by making use the solutions (24)-(27).

By using the constrained Hamiltonian structure presented, one can also proceed to perform operator quantization by expanding the generalized phase space variables in terms of normal modes. 


\section{References}

[1] A. Connes, Noncommutative Geometry, Academic Press, London, 1994.

[2] A. Connes, M. R. Douglas and A. Schwarz, JHEP 9802 (1998) 003.

[3] N. Seiberg and E. Witten, JHEP 9909 (1999) 032.

[4] L. Cornalba, D-brane physics and noncommutative Yang-Mills theory, hepth/9909081;

N. Ishibashi, A relation between commutative and noncommutative descriptions of D-branes, hep-th/9909176;

K. Okuyama, JHEP 0003 (2000) 016;

T. Asakawa and I. Kishimoto, JHEP 9911 (1999) 024;

B. Jurco and P. Schupp, Noncommutative Yang-Mills from equivalence of star products, hep-th/0001032;

$\mathrm{S}$. Terashima, On the equivalence between noncommutative and ordinary gauge theories, hep-th/0001111;

J. Madore, S. Schraml, P. Schupp and J. Wess, Gauge theory on noncommutative spaces, hep-th/0001203.

[5] H. B. Benaoum, Perturbative BF Yang-Mills theory on noncommutative $\mathbb{R}^{4}$, hep-th/9912036.

[6] E. S. Fradkin and G. A. Vilkovisky, Phys. Lett. B 55 (1975) 224;

I. A. Batalin and G. A. Vilkovisky, Phys. Lett. B 69 (1977) 309;

I. A. Batalin and E. S. Fradkin, Phys. Lett. B 122 (1983) 157.

[7] M. Henneaux and C. Teitelboim, Quantization of Gauge Systems, Princeton U. Press, Princeton, 1992. 